\title{
Gestión política del riesgo: reflexiones en torno a las regulaciones europea y catalana de $\mathrm{OMG}$
}

\section{Marina Di Masso Tarditti}

Universitat Autònoma de Barcelona. Institut de Ciència i Tecnologia (ICTA)

08193 Bellaterra (Barcelona). Spain

\section{Resumen}

Concebidos como «nuevos riesgos», los OMG agrícolas activan una dinámica política y social que tiene repercusiones sobre el proceso político-científico de toma de decisiones. Este artículo explora esta dinámica en un doble nivel, europeo y catalán. En el primer caso, el análisis se centra en la regulación de OMG, identificando los factores que explican su resultado final. En el segundo, el diagnóstico de la polarización del debate evidencia la existencia de procesos subyacentes, determinantes para la discusión. Estos procesos (lenguajes distintos y debate sobre el modelo agroalimentario) responden a la existencia de sistemas de valor contrapuestos y marcan la dinámica del conflicto.

Palabras clave: biotecnología agrícola, procesos político-científicos, gestión del riesgo, principio de precaución, coexistencia, modelo agroalimentario.

Abstract. The political management of risk: reflections on European and Catalan GMO regulations

Conceived as «new risks», agricultural GMOs activate a political and social dynamic with effects over the political-scientific decision-making process. This paper explores this dynamic in a double level, European and Catalan. In the first case, GMOs regulation is analysed, identifying the factors that explain its result. In the second case, the diagnosis of a polarized debate shows the existence of underlying processes, determinant for the discussion. These processes (different languages and debate on the agri-food model) are due to the existence of opposed value systems and they condition the dynamics of the dispute.

Key words: agricultural biotechnology, political-scientific processes, risk management, precautionary principle, coexistence, agri-food model.

\section{Sumario}

1. Introducción 4. La política europea de OMG:

2. La dinámica política y social de los precaución sin consumar

OMG

5. El caso catalán: coexistencia sin debate

3. Planteamiento de investigación

6. Conclusiones: un desajuste persistente Bibliografía 


\section{Introducción}

La gestión política de los organismos modificados genéticamente ${ }^{1}$ (OMG) agrícolas constituye un caso de estudio de interés particular, dada la multidimensionalidad del riesgo que estos productos entrañan. Más allá del ámbito agronómico (posibilidad de contaminación derivada de la coexistencia entre cultivos modificados genéticamente (MG) y no $\mathrm{MG}$ ), sanitario y ambiental (consecuencias sobre la salud de las personas y los ecosistemas), los riesgos asociados a la agricultura MG apelan a consideraciones más amplias, tanto de tipo ético (sobre el valor intrínseco de la vida), como económico (viabilidad de distintos modos de producción agraria), como político y social (definición, percepción y aceptabilidad del riesgo, delimitación de responsabilidades por daños eventuales). Éstas y otras repercusiones (de sostenibilidad, de relaciones entre el Norte y el Sur, de privatización del conocimiento) confieren a la política de transgénicos un interés normativo que otras políticas no tienen.

En un segundo ámbito, el interés por la regulación de OMG como objeto de estudio se justifica sobre la base teórica de los «nuevos riesgos» (Beck, 1998) de la modernidad ${ }^{2}$. La conceptualización de los OMG como tales les imbuye de una dimensión política que casa con la perspectiva ofrecida por las teorías de la gobernanza y el debate sobre la democratización del proceso político formal de toma de decisiones. Como elemento puente entre los nuevos riesgos y las nuevas formas de gobernanza, el principio de precaución es el paradigma en el que convergen ambas reflexiones. La discusión sobre el principio de precaución aparece en el planteamiento de una nueva gobernanza para gestionar el nuevo entorno de riesgo que, como aspecto de su complejidad creciente, caracteriza la época actual.

Asimismo, el estudio de las regulaciones europea y catalana (ésta como ejemplo particular del caso español) de OMG tiene una destacada relevancia empírica. La introducción y la comercialización de cultivos y alimentos MG en la Unión Europea (UE) ha sido y está siendo controvertida. Desde el primer momento, estas actividades han recibido un fuerte rechazo público por parte de ciertos sectores sociales, y en particular en algunos estados miembros, que forzaron la adopción de una moratoria en 1998 sobre nuevas autorizaciones. Por su parte, el caso catalán es particularmente interesante, dado que es una de las comunidades autónomas del Estado español (a su vez, el único estado miembro de la UE en que se cultivan OMG para su comercialización a gran escala) con más superficie de cultivos MG, una extensión que, además, va en aumento. Dada esta tendencia, actualmente existe una propuesta de Decret de

1. Los OMG son organismos cuyo material genético (ADN) ha sido modificado, no de manera natural por multiplicación o recombinación, sino mediante técnicas de ingeniería genética. Son conocidos comúnmente como «transgénicos».

2. La noción de riesgo, propia de la modernidad, «se origina con la comprensión de que resultados imprevistos pueden ser consecuencia de nuestras propias actividades o decisiones, en lugar de ser expresión de ocultos significados o de la naturaleza de las inefables intenciones de la divinidad» (Giddens, 2004: 40). 
Coexistència entre cultivos MG y no MG, una norma que captura la controversia sobre la agricultura modificada genéticamente en todas sus dimensiones, a través de las distintas definiciones que los agentes implicados hacen del problema.

Más allá de este triple interés, el debate alrededor de los OMG se enmarca en la relación entre ciencia, política y sociedad. En particular, en la redefinición del papel del conocimiento científico en la toma de decisiones políticas y en los cambios que empiezan a advertirse en la estructura social en reclamo de un espacio para la participación en esa toma de decisiones. Ambas dinámicas implican una redistribución de poder y funciones entre estas esferas y convergen, en última instancia, en el debate sobre la democratización de la ciencia y de la propia democracia.

El propósito de este artículo ${ }^{3}$ es mostrar cómo, a partir de su conceptualización como «nuevos riesgos», los OMG activan una dinámica política y social que tiene repercusiones sobre el ejercicio de toma de decisiones. Desde este punto de partida teórico, se describe la evolución del marco normativo de OMG a nivel europeo, señalando los factores que intervienen en este proceso y explican su resultado final. A partir de esta contextualización, el análisis de la controversia en Catalunya se centra en la identificación de los agentes implicados en la discusión y de los elementos que la articulan, mostrando cómo las posturas mantenidas sostienen un debate altamente polarizado como consecuencia de un enfrentamiento subyacente entre distintos sistemas de valor.

\section{La dinámica política y social de los $\mathrm{OMG}$}

En el tránsito de la sociedad industrial tradicional a la sociedad moderna concebida como «sociedad del riesgo» (Beck, 1998), cambian la tipificación, el origen y la percepción social del riesgo, pasando del predominio del riesgo exógeno, cuya génesis se entiende ajena al desarrollo económico y tecnológico, al predominio del riesgo endógeno ${ }^{4}$, cuyos conflictos asociados «estallan por causas sistemáticas que coinciden con el motor del progreso y la ganancia» (Beck, 1998: 46). En este tránsito, el aumento paralelo del reconocimiento social de los efectos secundarios del desarrollo como riesgos autoproducidos y de la desconfianza en la ciencia como fuente de soluciones, provoca como resultado una sensación generalizada de estar expuestos a riesgos que, por un lado, nos

3. El presente artículo es el resultado de una investigación más amplia en la que se ha abordado la gestión política del riesgo asociado a la agricultura transgénica en un doble contexto, europeo y catalán. Las páginas que siguen pretenden ofrecer un resumen ilustrativo de los principales resultados y conclusiones extraídas de la investigación. Di MASSO, Marina (2005): Gestión politica del riesgo. La controversia sobre la agricultura MG en Catalunya, en el marco de la regulación europea de transgénicos. Memoria de investigación. Doctorado en Sociología. UAB, septiembre.

4. O, alternativamente, riesgo "manufacturado», como aquél que es creado por el propio impacto de nuestro conocimiento creciente sobre el mundo, en contraposición al riesgo «externo», como aquél que se experimenta proveniente de las sujeciones de la tradición o la naturaleza (Giddens, 2005). 
son impuestos y, por otro, no podemos controlar, en lo que Beck describe como «la vivencia de estar afectado por un riesgo cerrado a la decisión» (1998: 47). A esta nueva tipología se ajustan los cultivos y alimentos MG.

\subsection{Nueva gobernanza para nuevos riesgos}

Ante este nuevo escenario se activa una dinámica social que se desdobla en dos procesos paralelos. Por un lado, la percepción de no control sobre los nuevos riesgos activa el reclamo por parte de la sociedad de un papel en su gestión. Por otro, ésta exige, asimismo, responsabilidades sobre su generación, ya que esos riesgos son percibidos, no como algo inevitable, sino como el producto de una decisión, es decir, de la elección de un determinado tipo de desarrollo y no otro. Ya no se identifica de manera automática progreso económico y/o científico con progreso social (Giddens, 2004), de manera que no se acepta cualquier avance técnico, ni sus consecuencias negativas asociadas, con el solo argumento de la mejora de la calidad de vida. A partir de este viraje fundamental, la dinámica política asociada a los nuevos riesgos supone la activación de la exigencia de responsabilidades por su autogeneración, entendiéndolos como las consecuencias no deseadas e impuestas de un tipo de desarrollo económico y tecnológico. En este sentido, la caracterización de los nuevos riesgos de la modernidad plantea el debate de fondo sobre qué sociedad se quiere $\mathrm{y}$, en el caso particular de la agricultura MG, qué tipo de modelo agroalimentario.

La reflexión sobre una nueva cultura política asociada a los nuevos riesgos gira en torno a la idea de que esta nueva tipificación sugiere formas de gobernanza nuevas ${ }^{5}$, puesto que, como producto de una simultánea construcción científica y social, los riesgos no pueden ser gestionados únicamente por aquéllos que los producen. Efectivamente, Kooiman (1993) define la gobernanza en términos de la interacción entre gobierno y sociedad y, como factores básicos de nuestra sociedad moderna, la complejidad, el dinamismo y la diversidad crecientes, originados por desarrollos sociales, tecnológicos y científicos, colocan a los sistemas de gobierno ante nuevos retos tales que se necesitan nuevas concepciones de gobernanza para gestionarlos.

El recurso al concepto de la nueva gobernanza por parte de las propias instituciones políticas hay que situarlo en el contexto de desafección democrática que afecta a las sociedades democráticas maduras e interpretarlo en clave de búsqueda de legitimidad de las decisiones en el seno del proceso político for$\mathrm{mal}^{6}$. Claramente, la controversia sobre la biotecnología contiene elementos

5. La idea de gobernanza se ha adoptado en distintas áreas y diversos contextos político-administrativos como una nueva manera de reflexionar sobre las capacidades del Estado y las relaciones entre el Estado y la sociedad. Para una revisión sobre las diferentes corrientes interpretativas sobre el fenómeno, consúltese Pierre y Peters, 2000.

6. Para una revisión bibliográfica sobre esta cuestión, véase una compilación de aportaciones de distintos autores en Hirst y Khilnani, 1996. 
de estos fenómenos de desafección y crisis de legitimidad políticas. Mazur (1981) caracteriza las controversias tecnológicas a través de diferentes factores, entre ellos que los principales participantes en la controversia son calificados de expertos en tecnología o ciencia, que los miembros del sector científico o técnico no controlan la disputa y, el punto relevante para el caso que nos ocupa, que ésta no está contenida en el proceso político formal de la democracia representativa, sino que la gente considera la cuestión lo suficientemente importante como para no dejarla exclusivamente en manos de las elites científicas o de los representantes electos. Se crean, así, grupos que desafían en última instancia la autoridad de los poderes públicos, objetando no sólo sus proyectos (en este caso, biotecnológicos), sino también su legitimidad y su habilidad para gestionarlos.

El debate alrededor de la biotecnología y los efectos de su aplicación no se plantea tanto en el terreno científico como en el plano de sus implicaciones sociales, económicas y culturales (Manzini y Bigues, 2000). En este contexto ampliado, y dado el nivel de incertidumbre asociado a los OMG, se manifiesta la necesidad de entablar un debate plural sobre estas consecuencias. La gobernanza aparece aquí como el concepto que invita a reunir las diversas posiciones que compiten defendiendo su propia visión del fenómeno y la definición que hacen del problema. Aquello que confiere interés a las nuevas formas de gobernanza es que suponen un cambio de enfoque desde uno unilateral (gobierno o sociedad civil separadamente) hacia uno interactivo. Un producto de la interacción e interdependencia en la elaboración de decisiones políticas es la propuesta de nuevas formas de gobernanza en las que los ciudadanos ejercen un control más directo sobre las decisiones políticas. Diferentes propuestas teóricas de refuerzo de la sociedad civil $^{7}$ giran alrededor de una mayor y mejor participación pública, que se erige como condición imprescindible para llevar a cabo verdaderos procesos de deliberación plural en torno a las preocupaciones sociales y las respuestas políticas a diseñar. A efectos de la gestión del riesgo, según De Marchi y Funtowicz (2004), en la discusión sobre nuevas formas de gobernanza va cobrando fuerza el argumento de que la seguridad sólo puede derivar de un diálogo abierto, de una participación amplia desde el comienzo y de una cooperación negociada entre todas las partes. Se hace necesario el establecimiento de un diálogo público sobre el cambio tecnológico y sus consecuencias problemáticas que permita reducir algunos de sus efectos más perniciosos (Giddens, 2005), siendo el caso de la agricultura $\mathrm{MG}$ paradigmático de esta necesidad.

\subsection{Nuevos enfoques para el proceso cientifico-politico}

La concepción de la ciencia como coproductora de los problemas que supuestamente debería solventar es una observación fundamental que apela a una

7. Desde la teoría de la democracia participativa, se pueden mencionar las aportaciones a cargo de Barber (1984) sobre democracia fuerte, Pateman (1970) sobre la función educativa de la participación y Etzioni (1991) sobre comunitarianismo (Font, 2001). 
necesaria visión integradora de las esferas científica, política y social en la que el reparto de poder existente entre ellas se va a ver alterado. Por una parte, en términos de la relación entre ciencia y sociedad, el hecho de que realidad y los riesgos estén científicamente producidos implica que la evaluación de éstos queda sujeta a una aceptabilidad social que supone admitir la existencia de factores que condicionan el ejercicio científico-técnico. Los juicios de valor y los diversos intereses asociados a esta actividad son condicionantes que es necesario explicitar a la hora de valorar la conveniencia de una opción frente a otra (en el caso que nos ocupa, tecnológica). Por otra parte, en términos de la relación entre ciencia y política, la falta de consenso científico sobre las consecuencias de la agricultura MG muestra que, en el ámbito de la gestión del riesgo, en algunos casos el conocimiento científico-técnico por sí solo no puede cumplir las expectativas de seguridad que pone en él la política. Por ejemplo, situaciones en que existe un elevado nivel de incertidumbre asociado al fenómeno en consideración y/o en que un público informado y sensibilizado cuestiona gestiones pasadas (como ocurre en el ámbito alimentario). La incertidumbre, la falta de evidencia empírica sobre posibles estados futuros del medio natural o la falta de consenso entre expertos son realidades actuales del ejercicio científico que limitan las estrategias tradicionales de gestión política ${ }^{8}$.

En el caso de la biotecnología, la evaluación del riesgo se centra principalmente en los efectos sobre la salud pública y el medio ambiente, pero el debate es, en efecto, más amplio. La introducción de OMG en la agricultura tiene implicaciones para la salud humana y para el entorno, pero también comerciales, de derechos de propiedad intelectual, de desigualdad entre el Norte y el Sur, ligadas a la coexistencia económicamente viable entre diferentes sistemas de producción agrícola, entre otras. Las implicaciones son múltiples y se discuten, en el fondo, los modelos de agricultura, de ciencia y de desarrollo deseados, y todo ello pasa por la incorporación de otros conocimientos (como la agroecología o la ética) al conocimiento experto que evalúa los posibles efectos de la tecnología y a que se recurre para tomar las posteriores decisiones políticas en la gestión del riesgo.

Siendo la ciencia, simultáneamente, fuente de solución y origen de problemas (Beck, 1998), ésta debe someterse a un proceso de redefinición en el

8. Estos casos en que se conjugan un elevado nivel de incertidumbre y un conocimiento científico limitado sobre las cuestiones fácticas y, a pesar de ello, se requiere tomar decisiones relativas a medidas de actuación, se ajustan al paradigma de la ciencia postnormal. $\mathrm{Al}$ abordar un problema, este método, desarrollado por Funtowicz y Ravetz, toma en consideración dos atributos: la incertidumbre y las apuestas de decisión. En función de los niveles de estos atributos, se generan tres estrategias posibles de resolución de problemas: la ciencia aplicada (los autores definen así la resolución tradicional de problemas), cuando ambos niveles son mínimos; el asesoramiento profesional, cuando alguno de los atributos es de grado medio y requiere cierto tipo de habilidad o juicio profesional, y la ciencia postnormal, cuando la incertidumbre es elevada y el nivel de politización del problema también (Funtowicz y Ravetz, 2000). Para una justificación del caso de los alimentos MG como ejemplo característico de ciencia postnormal, véase García Menéndez, 2004. 
que cobra un interés predominante el debate sobre cómo se desarrollan los resultados científico-técnicos, sobre qué tipo de ciencia se precisa para hacer frente a los efectos negativos de su desarrollo. Esta reflexión es relevante y muy pertinente en el caso de la biotecnología agrícola como actividad cuyas consecuencias en el largo plazo son mayormente desconocidas y de cuya gestión tenemos poca o ninguna experiencia histórica. Colocando el elemento de la incertidumbre en un lugar central, una redefinición del ejercicio científicotécnico centrada en la premisa de no generar irreversibilidades se traduce en una precaución sistémica que, en contraposición a la tradicional evaluación del riesgo caso a caso, asume un análisis ampliado que considera el contexto (de necesidades sociales e intereses económicos) en que se desarrollan la tecnología y sus aplicaciones, y su sostenibilidad a largo plazo?

Actualmente, en la gestión política de los riesgos convergen dos fenómenos asociados (De Marchi y Funtowicz, 2004). Por una parte, la existencia de regulaciones apoyadas en la ciencia que son problemáticas porque se basan en herramientas tradicionales de evaluación de riesgos que, dadas las actuales características de éstos (incalculables, impredecibles, irreversibles, globales) en la mayoría de ámbitos, actualmente son cuestionadas. Por otra parte, este cuestionamiento lo realiza una ciudadanía que está mejor educada y tiene acceso a una mayor información que en cualquier otra época anterior, lo cual le permite ser más crítica y exigente con sus gobernantes y con el uso que éstos hacen del conocimiento científico para tomar decisiones. En este contexto, los conceptos de gobernabilidad y riesgo quedan enlazados a través del principio de precaución, particularmente en el caso de desarrollos tecnológicos asociados a un elevado nivel de incertidumbre científica y con repercusiones potencialmente irreversibles. Como paradigma enfrentado a la evaluación tradicional de riesgos (Riechmann y Tickner, 2002), que da luz verde a nuevos productos siempre que no haya pruebas concluyentes de daño, el principio de precaución coloca la carga de la prueba en la inocuidad de los productos. Asimismo, subraya la consideración del principio de necesidad (social del nuevo producto) y la valoración de alternativas (menos inciertas y/o peligrosas), y sugiere que el desarrollo científicoproductivo, orientado de acuerdo con criterios socioecológicos, debe ser flexible y reversible. Como herramienta para manejar el aumento del riesgo manufacturado (Giddens, 2005), el principio de precaución ofrece un espacio y contempla una escala temporal adecuados para el control social de cambios tecnológicos de amplio alcance e impacto desconocido como es la agricultura MG.

\section{Planteamiento de investigación}

En términos operativos, la investigación aquí presentada se ha llevado a cabo a partir de los objetivos, las hipótesis y la metodología detallados a continuación.

9. Para una reflexión sobre la precaución sistémica aplicada al caso de los OMG, véase Tàbara y otros, 2003. 
Como objetivos principales, la investigación se ha propuesto:

a) Caracterizar la gestión política europea de OMG, en el marco general de un cambio de estrategia en la UE con relación a la gestión de riesgos alimentarios.

b) Determinar qué factores intervienen en los procesos de gestión política de los OMG y explican los actuales resultados, con el fin de explorar hasta qué punto existe una gobernanza distinta en la gestión de riesgos alimentarios en la UE.

c) Caracterizar la gestión política de OMG en Catalunya y compararla con el proceso de regulación europeo.

En el apartado precedente, se ha comentado la existencia actual de una voluntad institucional, particularmente a nivel europeo, de recuperar la confianza de la sociedad en las instituciones política y científica que se refleja en un discurso con continuas alusiones al concepto de gobernanza y a los elementos asociados de transparencia y participación. Centrándola en el caso de la regulación de OMG, la investigación ha tratado de explorar esta realidad a través de diversas hipótesis de trabajo, de las que, en este artículo, se destacan las siguientes:

- Las variables que influyen en el cambio en la gestión de riesgos alimentarios son de tipo sociopolítico: incremento de la incertidumbre, crisis alimentarias previas y necesidad de recuperar la confianza de la ciudadanía en las instituciones; y económico: asociadas al objetivo de competitividad en el sector biotecnológico ( $\mathrm{y}$ en el caso de la UE, a la necesidad de armonizar el mercado interno de OMG).

- Inscritos en una lógica de competitividad, los factores económicos tienden a ser más determinantes que los factores de tipo sociopolítico en la evolución del marco regulatorio de OMG.

- La controversia tecnológica asociada a los OMG captura cambios en la estructura social: una mayor presión de ciertos sectores sociales por participar en la toma de decisiones políticas.

La metodología seguida para el desarrollo de estos objetivos ha sido distinta según la escala geográfica. Para el caso europeo, los datos recogidos han provenido de fuentes secundarias de información, principalmente documentos elaborados por la Comisión Europea (CE). En el caso catalán, en cambio, la consulta de fuentes secundarias (a la que también se ha recurrido) ha sido, valga la redundancia, un elemento secundario.

La exploración de este segundo caso se ha basado en la explotación de fuentes de información primarias, escogiendo un método de investigación cualitativa (Denzin y Lincoln, 1994). Lo que se pretendía con el trabajo de campo (llevado a cabo durante junio y julio de 2005) era recoger las distintas definiciones del problema de los actores implicados en la controversia objeto de estudio, es decir, conseguir un tipo de información vinculado a los significados y 
a la interpretación que los individuos hacen de los riesgos de la agricultura MG y otras cuestiones asociadas a esta tecnología. Por esta razón, se ha considerado adecuado utilizar la técnica de la entrevista en profundidad, semiestructurada, individual y cara a cara (Corbetta, 2003). Este procedimiento permite desarrollar una conversación larga con las personas de la muestra de manera que vayan surgiendo los indicios de información antes mencionados. Efectivamente, la entrevista es un proceso comunicativo mediante el cual se puede "extraer» información de una persona, una información que se halla oculta en su biografía, esto es, en el conjunto de las representaciones asociadas a los acontecimientos vividos (Alonso, 1998).

Respecto a la presente investigación, la entrevista ha partido de un mínimo guión inicial de ámbitos temáticos preestablecidos (reproducidos a continuación), mediante el cual se han abordado los objetivos de la investigación a partir de las experiencias propias del entrevistado: presentación de la persona y de la institución a la que pertenece y su relación con los OMG, repaso de la llegada de los OMG a Catalunya (cultivos, normativa, etc.), beneficios y riesgos de la biotecnología agrícola (ventajas y desventajas), medidas a tomar, principio de precaución, expectativas de futuro de los OMG en Catalunya y relación con otros actores.

La elección del grupo de población a entrevistar se ha basado en la representatividad de los diferentes intereses implicados en el debate. Así, los actores de la muestra pertenecen a instituciones que inicialmente se clasificaron en «promotores» (Syngenta Seeds, SA), «afectados» (organizaciones agrarias: Unió de Pagesos, Joves Agricultors i Ramaders de Catalunya (JARC); organizaciones de consumidores: Organització de Consumidors i Usuaris de Catalunya, cooperativas de consumo ecológico y cooperativa de distribución de productos ecológicos, y organizaciones ecologistas: Associació Naturalistes de Girona, Ecologistas en Acción, Plataforma d'Entitats Ecologistes de Catalunya y Plataforma Transgènics Fora!) y «organismos institucionales» (Departament d'Agricultura, Ramaderia i Pesca $\left(D_{A R P}{ }^{10}\right)$ e Institut de Recerca i Tecnologies Agroalimentàries (IRTA $\left.{ }^{11}\right)$ ).

Una vez descrito el planteamiento de la investigación, en los apartados que siguen se presentan los principales resultados, haciendo una caracterización resumida de los dos casos de estudio abordados.

\section{La política europea de OMG: precaución sin consumar}

Diversos autores, desde diferentes perspectivas (Beck, 1998; Habermas, 1999; De Marchi y Funtowicz, 2004; Pindado, Rebollo y Martí, 2002), coinciden

10. El Gobierno autónomo catalán (la Generalitat de Catalunya) regula los sectores agroalimentario y pesquero a través de este Departamento.

11. El IRTA es una empresa pública de la Generalitat que centra su actividad en la investigación científica y la transferencia tecnológica en el ámbito de la agricultura, la acuicultura y la industria agroalimentaria. 
en señalar que vivimos en una época de crisis de legitimidad de las instituciones. Particularmente relevante es el caso del conocimiento científico y la democracia representativa. En este contexto, la gobernanza es el concepto del que se sirve la Comisión Europea para intentar recuperar la confianza de la ciudadanía europea, especialmente en cuestiones ligadas a la seguridad alimentaria, ámbito en que reaparece el debate sobre el papel del conocimiento experto en la toma de decisiones políticas. La pérdida de confianza en este ámbito es especialmente acusada en la UE, debido a que, en determinados casos en el pasado, conocimiento científico y representantes electos han actuado respondiendo a intereses distintos del interés general ${ }^{12}$. En respuesta a este descrédito, la CE (2001b) ha producido el Libro Blanco sobre Gobernanza Europea y el Libro Blanco sobre Seguridad Alimentaria (1999). Asimismo, la Comisión (CE, 2001c) ha elaborado un documento sobre cuándo y cómo usar el principio de precaución ${ }^{13}$ en el análisis (esto es, en la evaluación, gestión y comunicación) del riesgo, con el fin de hacerlo operativo.

\subsection{El contexto regulatorio}

Las sucesivas crisis alimentarias que se han dado en la UE han acarreado consecuencias sobre la opinión pública europea, tanto en términos de impacto en la percepción social del riesgo biotecnológico, como de desconfianza en las instituciones responsables de la gestión política del riesgo alimentario. Efectivamente, la CE (2001a) asegura en el documento de consulta de su comunicación Hacia una visión estratégica de las ciencias de la vida y la biotecnología, que las distintas crisis que en los noventa minaron la confianza del consumidor en la seguridad de la cadena alimentaria, remarcando la crisis de las vacas locas y la contaminación de pollos por dioxinas, han consolidado un cambio de orientación de las políticas públicas en el ámbito de la alimentación y han reforzado normativas y criterios de seguridad, tanto para alimentos de consumo humano como para piensos animales. Como muestra de este nuevo marco europeo de análisis del riesgo, además de publicar su Libro Blanco sobre Seguridad Alimentaria, la CE creó posteriormente la Autoridad Europea de Seguridad Alimentaria (EFSA, por sus siglas en inglés), motivada por la necesidad de abordar la cuestión de la confianza de los consumidores internos y de los socios comerciales externos en el campo del suministro de alimentos. Uno de sus ocho paneles científicos está dedicado específicamente a los OMG.

12. En Harremoës y otros (2002) puede leerse un capítulo con información detallada sobre la gestión de la crisis de las vacas locas por parte del Gobierno británico, un ejemplo grave de manipulación y ocultamiento de la información por parte de las instituciones científicas y políticas.

13. En su Comunicación sobre el principio de precaución, la CE establece una guía de principios generales para su aplicación. Entre otros, se menciona la revisión de desarrollos científicos, que establece que «las medidas [precautorias], aunque provisionales, deben ser mantenidas mientras los datos científicos sean incompletos, imprecisos o no concluyentes y mientras el riesgo se considere demasiado elevado para ser impuesto a la sociedad». 
La experiencia concreta de los mencionados episodios de crisis ha tenido dos efectos claros. Por una parte, en términos de percepción social del riesgo, ha acentuado la desconfianza del público en el papel del conocimiento científico en la toma de decisiones políticas. Por otra, en términos de gestión política del riesgo, ha provocado la recurrente explicitación en el discurso de la Comisión de una voluntad de transparencia y participación pública en la comunicación y gestión del riesgo (alimentario). A nivel institucional, en el primer caso, la experiencia se ha traducido en la manifestación de una voluntad de no esconder las incertidumbres asociadas a la tecnología y al eventual impacto de sus aplicaciones. En el segundo, ha conllevado la toma de una actitud de prudencia respecto a nuevas autorizaciones comerciales de OMG. Esta actitud se aprecia en la consideración del principio de precaución en la regulación de OMG desde el inicio de su diseño, cosa que supone una diferencia cualitativa y una novedad respecto a los anteriores casos de gestión de riesgos alimentarios.

\subsection{Proceso: fases y variables}

El marco regulatorio de los OMG agrícolas en la UE, teniendo que integrar sensibilidades muy diferentes, está influido básicamente por dos factores. Un primer factor es económico y está asociado al desarrollo del potencial competitivo de la UE en el sector biotecnológico. El segundo factor es de carácter sociopolítico, vinculado a la percepción social negativa de los OMG y a un sentimiento generalizado de desconfianza en la ciencia y la política en la gestión de temas de seguridad alimentaria entre la sociedad europea ${ }^{14}$.

En la elaboración de la política europea de OMG (ver gráfico 1), pueden identificarse hasta la fecha tres grandes fases, delimitadas a su vez por dos cambios. Siguiendo una tendencia precautoria creciente, esta evolución tiene una etapa preliminar previa a 1990, en la que la regulación de la agricultura MG es inexistente. Durante esta etapa, no existen cultivos MG para su comercialización en la UE, pero sí cultivos experimentales.

1. Marca el inicio de la primera fase (1990-1998) la aprobación de la Directiva 90/220/CEE, sobre la liberación experimental y la comercialización de OMG. El objetivo principal de esta directiva era el establecimiento de un mercado interno europeo armonizado para el libre cultivo y comercialización de OMG. En términos de la antes mencionada reorientación en la

14. Para sondear la posición de la opinión pública europea respecto a los transgénicos, consúltense los eurobarómetros EB 35.1 (1991), EB 39.1 (1993), EB 46.1 (1996), EB 52.1 (1999), EB 58.0 (2002) y EB 64.3 (2006) en el enlace de página web de la UE: http://europa.eu.int/comm/public_opinion En esta misma página, también pueden consultarse dos informes especiales: Europeans, Science and Technology y Social Values, Science and Technology, ambos en el marco del EB 63.1 de 2005. 
Gráfico 1. Esquema de proceso de la regulación europea de OMG agrícolas

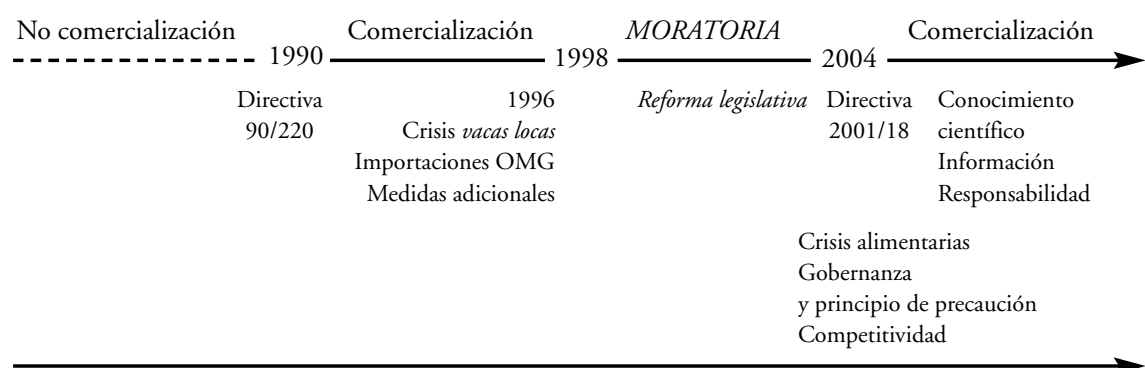

PRECAUCIÓN

Fuente: elaboración propia.

estrategia de gestión del riesgo alimentario de la UE, la elaboración de esta primera directiva representó un intento innovador de combinar los avances científicos y comerciales con la precaución ${ }^{15}$ (Carr, 1999). Sin embargo, se quedó corta en esta tentativa. Como principal carencia, la norma no contemplaba medidas precautorias para todo el ciclo de vida del producto, asumiendo que la aprobación de un OMG para su comercialización implica que, una vez obtenida esta autorización, un cultivo es seguro de ahí en más y no requiere más supervisión. De este modo, dejaba a cada estado miembro la responsabilidad de evitar eventuales efectos adversos de estos organismos. A nivel institucional, esta delegación se tradujo en la manifestación de posturas diversas en el seno de la Unión respecto a la evaluación del riesgo de los OMG y la severidad de las medidas precautorias a aplicar para controlar esos efectos eventuales. Actuaciones contradictorias entre decisiones de la CE y actitudes reticentes de algunos estados miembros (que más adelante forzarán una moratoria sobre cultivos MG), así como del Parlamento Europeo $(\mathrm{PE})^{16}$, mostraron que la regulación de OMG vigente no estaba satisfaciendo el objetivo marcado de armonización normativa. Ante los problemas de implementación derivados de esta situación, se dieron algunos pasos para mejorar el marco regulatorio, estableciéndose una serie de medidas precautorias adicionales para la fase de comercialización que inicialmente la Directiva no contemplaba (a asumir de manera voluntaria por las industrias agroalimentaria y tecnológica, relativas a cuestiones de monitoreo ambiental, protocolos de cultivo y guías de buenas prácticas, y esquemas de etiquetado). A pesar de estas reformas,

15. Sobre el principio de precaución en la regulación europea de transgénicos, véase Tait, 2001.

16. En la controversia sobre los OMG, el Parlamento europeo a menudo defiende una postura más precautoria que la que mantiene la Comisión, más cercana a los intereses de la industria biotecnológica. 
dado el contexto adverso existente (tanto social, con una opinión pública sensibilizada, como político, con importantes discrepancias entre estados miembros), se forzó finalmente una revisión al alza, en términos precautorios, de la normativa hasta ese momento vigente de OMG.

2. Este proceso se concreta en el establecimiento, en 1998, de una moratoria de facto sobre nuevas autorizaciones de eventos biotecnológicos ${ }^{17}$, elemento de inflexión que inaugura la segunda fase (1998-2004) de la política europea de transgénicos ${ }^{18}$. La moratoria fue promovida por una serie de estados (Francia, Grecia, Italia, Dinamarca, Luxemburgo, Bélgica y Austria) argumentando la necesidad de disponer de más información sobre los efectos de los OMG antes de aprobar nuevos eventos, sobre la base de amenazas para la salud y el entorno.

Como antecedentes de esta inflexión, las manifestaciones en contra de las primeras importaciones de OMG (soja y maíz $\mathrm{Bt}^{19}$ ) que llegan en 1996 al puerto de Barcelona procedentes de EEUU ${ }^{20}$, visibilizaron una postura de rechazo a estos cultivos por parte de amplios sectores de la opinión pública europea, y la atención mediática que se prestó a los acontecimientos contribuyó a potenciar el debate sobre la agricultura MG y sus implicaciones, reforzando la variable de la aceptabilidad social. Como un nuevo eslabón de una larga cadena de peligros asociados a la alimentación, la llegada de los transgénicos a Europa despertó nuevamente preocupaciones en torno a la cuestión de la evaluación del riesgo y el principio de precaución.

Más allá de las objeciones relativas a la falta de consenso científico sobre los eventuales efectos adversos de los OMG, el objetivo económico de competitividad en el sector biotecnológico fue el elemento guía de las iniciativas de la CE en este terreno ${ }^{21}$. Puesto que los defectos legales y problemas políticos de la Directiva estaban retrasando nuevas autorizaciones, en este contexto de moratoria a la CE y a la industria agrobiotecnológica europea también les convenía una revisión, con el objetivo último de conseguir una base de consenso tal que, una vez aprobado un OMG, los estados miem-

17. Los nuevos eventos son las modificaciones genéticas que contienen las nuevas variedades para las que se pide autorización.

18. Los cultivos experimentales son una cuestión aparte. Las pruebas de campo se han seguido desarrollando y, de hecho, son los cultivos que pueden consultarse (localización, extensión, etc.) (en la página web del Joint Research Centre, http://gmoinfo.jrc.it), no así los cultivos comerciales.

19. Las siglas Bt son una abreviación de Bacillus thuringensis, una bacteria con propiedades insecticidas que se introduce mediante manipulación genética en el maíz que de ella recibe el nombre.

20. A raíz de la crisis de las vacas locas y la supresión de las harinas animales en los piensos, la UE pasó a importar forraje transgénico de EEUU y Argentina, los dos mayores productores mundiales de este producto.

21. En la llamada Estrategia de Lisboa (adoptada en el Consejo Europeo de Estocolmo en marzo de 2001), la Comisión Europea establece la competitividad en el sector de la biotecnología como objetivo estratégico de la UE para su desarrollo económico. 
bros acataran la decisión en lugar de manifestar objeciones o imponer controles adicionales ${ }^{22}$.

3. El proceso de reforma legal culmina con la aprobación de la Directiva 2001/18/CE, cuyo objetivo es articular un marco regulatorio más estricto que tranquilice a la opinión pública, incorporando nuevas y más severas medidas de control. La nueva directiva contempla la rastreabilidad de los OMG en la cadena alimentaria, da seguimiento a los OMG liberados al entorno estableciendo registros públicos de cultivos comerciales y crea además un sistema de etiquetado de alimentos exigente que, entre otras medidas, obliga a etiquetar todo alimento que contenga ingredientes MG (a partir de un $0,9 \%$ de presencia). Su aprobación supuso el fin de la moratoria e inauguró una tercera fase (de 2004 en adelante), en la que nos encontramos actualmente, de libre circulación de OMG en la UE.

\subsection{Resultado: producto final y elementos para la controversia}

El cambio a subrayar respecto a la norma precedente es que la nueva directiva se dirige al proceso de creación del producto y no al producto en sí, lo que la caracteriza como una regulación horizontal. Más allá del control del resultado final (los productos biotecnológicos), el proceso que lleva a su creación es objeto de un control preciso y medido (Dorsey, 2002, citado en Riechmann, 2004), lo cual le confiere un carácter estratégico que, en principio, garantiza una regulación más estricta y transversal.

Sin embargo, a pesar de la introducción de mayores controles de los OMG en todo su ciclo de vida, existen importantes objeciones a la nueva directiva que permiten cuestionar el grado real de protección brindado. Son cuestiones relevantes ligadas al sistema de etiquetado, que la actual normativa deja incompleto (no obliga a etiquetar carne ni otros derivados de animales alimentados con OMG, una remarcable deficiencia, puesto que la cabaña ganadera es gran consumidora de piensos y forrajes, en su mayor parte MG), y que resulta todavía ambiguo en la definición de lo que se considera un producto MG y lo que no; al registro de la información pública sobre los cultivos MG (no son accesibles los datos de localización y extensión de las parcelas de cultivos con fines comerciales), poniendo en entredicho el discurso sobre transparencia, y a la delimitación de responsabilidades por parte de las empresas agrobiotecnológicas (todavía está pendiente una legislación que delimite claramente la responsabilidad civil en caso de contaminación genética, dejando desamparados a los posibles afectados por daños derivados del uso y cultivo de OMG). Directamente relacionado con este punto, permitir la coexistencia entre culti-

22. Efectivamente, la CE considera que «la incertidumbre sobre la aceptabilidad social ha contribuido a distraer la atención [...] de los factores que determinan nuestra capacidad para la innovación y el desarrollo tecnológico [...]. Ello ha anquilosado nuestra posición competitiva, debilitado nuestra capacidad de investigación y podría limitar nuestras opciones políticas en el largo plazo» (CE, 2002). 
vos MG y no MG cuando no existe todavía delimitación legal de responsabilidades en caso de daños por contaminación es una política de hechos consumados que, por un lado, es contraria a la lógica precautoria y, por otro, limita el debate público sobre la tecnología y sus impactos, poniendo en entredicho también el discurso de participación y nueva gobernanza planteado en el sentido de responsabilidad compartida.

\section{El caso catalán: coexistencia sin debate}

Si bien en el Estado español las comunidades autónomas tienen transferidas las competencias de agricultura, las disposiciones de estas regiones en el ámbito de los OMG deben obedecer a normas de rango superior. La normativa europea ha sido transpuesta a nivel estatal (tanto la Directiva 2001/18 como sus modificaciones posteriores, así como los reglamentos que regulan el etiquetado y la rastreabilidad a lo largo de la cadena alimentaria). Una vez el Estado aprueba una nueva variedad para su comercialización, las comunidades gestionan el cómo, es decir, las condiciones de cultivo, así como los efectos de este cultivo comercial, lo cual incluye diseñar una regulación de coexistencia entre cultivos MG y no $\mathrm{MG}$, con el fin de garantizar la viabilidad de todos los modelos productivos.

Respecto al control de la producción agrícola MG en Catalunya, la norma que constituirá la piedra angular de la regulación de esta actividad es el Decret de Coexistència (en adelante Decret), que, en el momento de redactar estas líneas (septiembre 2006), todavía no ha sido aprobado por el gobierno catalán, si bien se halla en la última etapa previa a su publicación definitiva. En cuanto a antecedentes, existe un precedente inmediato al Decret a nivel estatal. A finales de la legislatura pasada (febrero de 2004), el gobierno del Partido Popular elaboró un borrador de Real Decreto de Coexistencia inspirado en las Recomendaciones europeas $^{23}$ cuya tramitación fue paralizada en la siguiente legislatura, con el PSOE en el poder, con lo cual se reinició un periodo de reelaboración. La última versión de este Real Decreto (20 de abril de 2006) se sometió a alegaciones el pasado junio (2006) y se espera su aprobación próximamente.

En Catalunya se cultivan diferentes variedades, de diferentes empresas, de maíz transgénico Bt (exclusivamente para la fabricación de pienso animal) desde 1996. Sin embargo, la iniciativa de elaboración del Decret no surge hasta 2003, respondiendo a la necesidad de regular un sector que se está disparando ${ }^{24}$. La discusión de los sucesivos borradores ha puesto sobre el tablero diversas

23. Sobre la cuestión de la coexistencia, la Comisión Europea (CE, 2003) ha elaborado un documento guía de recomendaciones destinado a asesorar a los estados miembros. Más allá de estas recomendaciones no vinculantes, no existe normativa comunitaria alguna relativa a esta cuestión, puesto que, en aplicación del principio de subsidiariedad, su regulación corresponde a cada estado.

24. La superficie cultivada con maíz MG en Catalunya crece exponencialmente: pasó de 5.390 ha en 2003 a 16.259 ha en 2005 . Esto supone un $13,15 \%$ y un $39,90 \%$, respectivamente, de variedades MG sobre el total del maíz cultivado. Fuente: DARP. 
cuestiones asociadas a la agricultura MG que van más allá de los aspectos puramente técnicos, elementos que desempeñan un papel central para entender la polarización que caracteriza la relación entre las posturas anti y pro-OMG.

La clasificación de los actores implicados en la controversia en estos dos bloques se desprende del trabajo empírico llevado a cabo durante la investigación. Inicialmente (véase apartado 3, más arriba), se clasificaron los actores en tres grupos, sin embargo, a la luz de las informaciones recogidas, se estimó más conveniente agrupar a los actores en dos bloques, añadiendo a los "promotores» los organismos institucionales (DARP e IRTA) y JARC, y agrupando en un grupo de «detractores» al resto de actores. Esta reclasificación captura mejor el estado de la cuestión en Catalunya en cuanto a posturas ante la agricultura MG y avanza ya la polarización como característica del debate.

Como foto fija del análisis del caso catalán, el diagnóstico de la polarización evidencia la existencia de diferentes procesos subyacentes en la discusión: lenguajes diferentes y enfrentamiento sobre el modelo productivo, y confusión sobre el principio de precaución y obsolescencia del proceso político-científico tradicional de toma de decisiones. Estos procesos subyacentes responden, en primera y última instancia, a la existencia de sistemas de valor distintos asociados a las posturas enfrentadas, que marcan la dinámica del conflicto. Más allá de la discusión sobre las características de la tecnología y sus aplicaciones, otros autores han constatado esta dicotomía de fondo en el debate sobre la ingeniería genética. Como condicionante de las opiniones mantenidas y de las acciones emprendidas por cada bloque, se da un enfrentamiento de fondo entre lo que algunos llaman «cosmovisiones distintas» (Todt, 2004) y otros «racionalidades contrapuestas» (Muñoz, 1997). La constatación de esta dicotomía explica la existencia de posiciones prácticamente inamovibles entre las que no parece haber puntos de convergencia ${ }^{25}$.

\subsection{El detonante de la controversia}

El proceso de regulación del sector a través del Decret ha sacado a la luz las distintas cuestiones asociadas a la práctica de la agricultura transgénica, lo cual

25. Respecto al contexto del debate en Catalunya, todos los actores entrevistados (tanto promotores como detractores) coinciden sin excepción en admitir la existencia de una opinión pública contraria a los OMG, apuntando diversas explicaciones: la población no percibe beneficios directos para el consumidor, no asocia "transgénico» a "calidad alimentaria», está sensibilizada por la experiencia de pasadas crisis alimentarias y mantiene cierta desconfianza hacia los avances científicos. Más que dar por sentada la existencia de esta opinión pública generalizada, sería más prudente y ajustado a la realidad hablar en términos de tendencia. Si bien no hay datos que, a nivel catalán, confirmen la existencia de este clima desfavorable, el Eurobarómetro de 2005 muestra que en España el apoyo a los alimentos MG es de un 34\% (sólo 7 puntos por encima de la media europea). El CIS (estudio 2412) recoge también la postura reticente de la sociedad española respecto a las aplicaciones de la biotecnología en la agricultura y la alimentación, a pesar de que el 54,3\% y el 26,5\% de los encuestados se declaran "poco» $\mathrm{y}$ «nada informados» sobre esta tecnología. 
ha obligado a los diferentes sectores implicados a posicionarse, implícita o explícitamente, sobre la controversia. El objetivo de la norma es garantizar la libertad de elección del agricultor, de cultivar siguiendo el tipo de producción que desee (y del consumidor, de adquirir o no productos MG). El documento ha recibido fuertes críticas por parte de ciertos sectores sociales, centradas fundamentalmente en sus contenidos, pero también referentes al proceso mismo de elaboración seguido por el DARP, a quien se le reprocha su poca permeabilidad.

En cuanto al contenido, los argumentos esgrimidos por el bloque detractor para oponerse al Decret son diversos: los mecanismos contemplados de control de cultivos y semillas MG son ineficaces e insuficientes, la responsabilidad en caso de contaminación genética queda parcialmente indefinida, y no se asignan recursos para garantizar un satisfactorio desarrollo y cumplimiento de la norma. Más allá de su contenido, también respecto al proceso político de elaboración del Decret, las posiciones están polarizadas. Desde su postura en solitario, el responsable entrevistado del DARP defiende que el proceso ha sido abierto, inspirado por un principio de transparencia. En contra, el resto de actores coinciden en que las observaciones que han realizado no han sido tenidas en cuenta y, en este sentido, valoran que no ha existido negociación. La ausencia de control social y de debate es un argumento del discurso anti-OMG identificado también en otras investigaciones ${ }^{26}$. En general, la crítica no se centra en el grado de apertura del proceso de elaboración, sino que se niega que aquélla haya sido una característica del proceso desde su inicio y lo que se cuestiona esencialmente es la calidad de la participación. El resultado final es un documento que sólo desde el DARP se defiende abiertamente (el IRTA en esta cuestión se desmarca de éste, al estimar que la norma se ha elaborado "de espaldas al campo»). En cuanto a la gestión del conflicto de fondo entre cosmovisiones diferentes, el caso catalán se ajusta a lo que Todt (2004) caracteriza como un escenario de imposición, en contraposición a un escenario de negociación. Según el autor, el resultado de esta estrategia es el fortalecimiento de los actores sociales críticos con la tecnología.

\subsection{Dos lenguajes distintos}

En el debate sobre la agricultura MG en Catalunya, los discursos que mantienen las dos posturas enfrentadas son de naturaleza diferente: técnico el de los defensores, político (también) el de los detractores. Simplificando, se puede decir que se responde a cuestiones técnicas con argumentos políticos y a argumentaciones políticas con cuestiones técnicas. Se ha señalado en otros trabajos ${ }^{27}$ cómo los promotores de la biotecnología atribuyen a los detractores una confusión entre cuestiones político-ideológicas y argumentos propiamente cien- 
tíficos para reforzar su postura y desacreditar la contraria. Este desencuentro entre marcos argumentativos genera una situación que desestimula el debate, cuando no lo anula, con lo que queda reducido a un intercambio de opiniones ya conocidas. Veamos a continuación los posicionamientos que delimitan el enfrentamiento.

A nivel sustantivo, los dos bloques articulan su discurso ofreciendo lecturas diferentes sobre las implicaciones del desarrollo de la biotecnología y sus productos. Los defensores de los OMG agrícolas centran su argumentación en los beneficios de la tecnología y en la posibilidad de tener conocimientos suficientes para garantizar su seguridad. Los detractores, en cambio, subrayan la existencia de un elevado grado de incertidumbre sobre las consecuencias de esta actividad para la salud y el medio ambiente. Ambos grupos difieren en primera y última instancia en el juicio que emiten sobre la posibilidad de conocer y controlar los riesgos que comportan los OMG. En consecuencia, un grupo identifica comercialización con seguridad y el otro, con temeridad. En cuanto a las ventajas, los promotores subrayan el beneficio de un sector concreto (beneficio económico, de los agricultores que cultivan maíz MG) al que los detractores oponen un riesgo multidimensional (ambiental, sanitario, socioeconómico) que asume la sociedad en su conjunto. Las cuestiones de regulación y control, incertidumbre y seguridad, y la discusión sobre los eventuales beneficios de la biotecnología son elementos centrales en la controversia en Catalunya y, sumando el del acceso a la información, se corresponden con las preocupaciones comunes en el debate a nivel europeo desde sus inicios ${ }^{28}$.

La polarización de la controversia responde al hecho último de que una postura («anti») sitúa el desarrollo de la tecnología en un determinado contexto (social y económico), mientras que la otra ("pro») obvia o desplaza la atención de este contexto para centrarse en la tecnología y sus productos en sí mismos. Éste es el eje principal que articula la polarización: se enfrentan un análisis del riesgo centrado en el contexto a un análisis centrado en el producto en el que la cuestión es si se puede prescindir o no del marco (de necesidades sociales e intereses económicos) en el que se desarrolla la tecnología. El reclamo de los anti-OMG por la toma en consideración del contexto en que se enmarca el desarrollo de la biotecnología apela automáticamente al debate sobre el modelo agrícola (e, indirectamente, alimentario) como telón de fondo. Se vehicula a través del rechazo a los OMG una oposición al modelo agrario industrial heredero de la Revolución Verde, del cual la actual revolución biotecnológica sería el escalón más reciente y novedoso. Cuando los actores antiOMG en Catalunya denuncian una connivencia del DARP con los intereses empresariales de la biotecnología, se están refiriendo a la promoción conjunta de un determinado modelo por parte de estos dos actores. Así, en la cuestión

28. Véase el informe elaborado por LEMKOW, L. (1993): «Public attitudes to genetic engineering: some European perspectives», European Foundation for the Improvement of Living and Working Conditions. 
sobre la existencia de intereses por regular en un sentido permisivo con la agricultura MG, arraiga necesariamente la discusión sobre el modelo agrario.

\subsection{El debate subyacente}

El impulso que están recibiendo los transgénicos en Catalunya se inscribe en la apuesta del DARP por un modelo de profesionalización y empresarialización del campo, un modelo en el que la introducción de nuevas tecnologías es fundamental (Peix, 2004). Dentro de este esquema, la biotecnología desempeña un papel estratégico, necesario según los actores pro-OMG para la dinamización del sector y que, según los actores anti-OMG, condena a los agricultores a incrementar su dependencia de las empresas agrobiotecnológicas, cuando no a desaparecer.

La identificación del gobierno catalán y la industria biotecnológica como un tándem es un lugar común en la crítica de los actores anti-OMG, quienes a este tándem añaden la ciencia oficial (universidades e IRTA ${ }^{29}$ ) en la promoción del modelo transgénico. Como resultado, los críticos utilizan el argumento del vínculo de las instituciones públicas de gobierno e investigación con las empresas biotecnológicas para cuestionar las decisiones de las primeras y desacreditar los resultados ofrecidos por las segundas. La variable de la confianza en las instituciones (políticas y científicas) desempeña un papel importante en la postura crítica con la biotecnología agrícola, así como en la creación de un clima desfavorable a sus productos extendido entre la opinión pública.

Para los promotores de los OMG, el contexto socioeconómico en el que se desarrolla la biotecnología es una variable coyuntural en la controversia, mientras que sus detractores lo conciben como un elemento central del cual no se puede prescindir, al discutir las implicaciones de los productos y aplicaciones de esta tecnología. Por lo tanto, existe un desajuste primero y último sobre la localización y el contenido del debate. Haciendo una analogía poco sutil pero gráfica, se plantea la pregunta de ¿qué es primero, el modelo (económico-productivo, agroalimentario) o el producto transgénico?

\section{Conclusiones: un desajuste persistente}

La investigación realizada permite concluir que la controversia sobre la agricultura MG en Catalunya contiene elementos comunes al debate que, a nivel europeo, enfrenta a defensores y detractores de la agrobiotecnología, permitiendo caracterizar una controversia global desde un análisis local. Por una parte, en relación con la regulación de OMG como política pública, tanto el

29. La fuente entrevistada del IRTA comentaba la necesidad que tiene este organismo público de establecer convenios con empresas para poder financiar su actividad investigadora y, al mismo tiempo, advertía sobre la no conveniencia de esta práctica para poder mantener la independencia en el libramiento de resultados y que éstos no sean capitalizados por las empresas. 
caso europeo como el catalán son producto de un balance desequilibrado entre los factores económico y sociopolítico, siendo el primer factor más determinante que el segundo. Por otra, en la controversia en Catalunya existe un enfrentamiento básico entre narrativas de naturaleza distinta y diferentes aproximaciones a la gestión del riesgo, directamente relacionadas con la defensa de modelos productivos y de sociedad diferentes. Subyacen a los discursos enfrentados sistemas de valor distintos que condicionan las lecturas que ambas posturas hacen de la actividad biotecnológica, lo cual determina actuaciones y opiniones. Efectivamente, la idea de que las reacciones de las personas a la información de que disponen está influida por sus creencias previas, es una idea bien arraigada en el ámbito de la psicología social (Poortinga y Pidgeon, 2006). En contextos como el catalán, altamente polarizados, las actitudes fuertemente positivas y negativas tienen una gran probabilidad de mantenerse estables en el tiempo, de tener un impacto sobre los comportamientos y el procesamiento de la información, y de ser resistentes a la persuasión (Petty y otros, 2005).

El marco legal de OMG en la UE obedece en gran medida a la necesidad de armonizar criterios regulatorios para consumar el mercado interno de OMG. Supeditado a éste, se hallaría el objetivo de resolución de aspectos de carácter social y político, relacionados con la protección de la salud y el derecho a la información del consumidor ${ }^{30}$. Persiste un desequilibrio entre consideraciones económicas de rentabilidad y competitividad, y consideraciones de «segundo orden», entre las cuales cuestiones de participación y gobernanza aparecen más como elementos de legitimación de decisiones ya tomadas (de ahí que se favorezca $^{31}$ la coexistencia, contradictoria con el principio de precaución reivindicado por la propia CE y con la libertad de elección de opciones de producción) que como principios guía de un debate realmente abierto.

En este contexto de desequilibrio entre intereses, el caso catalán evidencia la necesidad de que la Administración que regula los diferentes modelos productivos clarifique su postura sobre el modelo transgénico. Se manifiesta la necesidad de resolver la contradicción existente entre una pretensión discursiva de neutralidad y una defensa fáctica de la coexistencia entre cultivos, dada la controversia sobre la agricultura MG y sus implicaciones como modelo que puede ser incompatible, y de manera irreversible, con otros tipos de producción, especialmente la ecológica, y que cuenta con una opinión pública desfavorable. Ello pasaría por establecer una delimitación clara de la finalidad de la regula-

30. El Consejo Europeo de Estocolmo (2001) invitó a la Comisión y al Consejo europeos a «fortalecer la competitividad del sector biotecnológico europeo para alcanzar a los competidores líderes mientras se asegura que esos desarrollos ocurren de manera sana y segura para los consumidores y el entorno» (CE, 2002) (cursiva mía).

31. La coexistencia se favorece en la medida en que, si bien no se obliga a nadie a cultivar transgénicos, a nivel institucional la coexistencia no se cuestiona. Es más, las recomendaciones europeas hablan de regular en el sentido de minimizar la contaminación, lo cual implica aceptar que van a existir perjuicios derivados de esta opción de convivencia entre distintos sistemas productivos. 
ción de los OMG como política pública: proteger la salud humana y medioambiental garantizando la viabilidad de todos los sistemas productivos es un objetivo que no parece poder compatibilizarse de manera equilibrada con la apuesta por la biotecnología en el sector agrícola (en este ámbito, el DARP sigue la línea marcada por la CE). La pregunta por qué objetivo prima, conlleva en su respuesta la defensa de un determinado modelo agrario. En relación con este asunto, la insistencia de los promotores en gestionar la tecnología haciendo hincapié en su neutralidad es precisamente un factor que fortalece a la oposición (Todt, 2004), lo cual contribuye a dificultar el diálogo.

Por otro lado, si bien es cierto que los detractores de la biotecnología agrícola vehiculan, a través de su rechazo, una crítica de carácter más estructural, parece igualmente acertado afirmar que este vínculo no se puede obviar en la discusión sobre sus implicaciones. El debate lleva imbricadas cuestiones económicas y sociopolíticas que, exploradas en profundidad, desembocan en un replanteamiento del proceso político-científico de toma de decisiones y, ligado a éste, de la gestión tradicional del riesgo. El caso de la agricultura MG pone de relieve que la variable de la aceptabilidad social tiene un papel a desempeñar en el desarrollo de resultados científicos y en la evaluación de los riesgos que generan. La voluntad de participación que manifiestan determinados actores sociales remite a la definición del nivel socialmente aceptable de riesgo como un proceso de codefinición de carácter político, extrayendo los OMG del sustrato científico-técnico en el que se hallan ceñidos actualmente. Efectivamente, para desbloquear el debate hace falta ampliarlo (incorporando otras dimensiones más allá de la estrictamente científico-técnica en la evaluación del riesgo y a otros actores en su gestión) y relocalizarlo (¿qué modelo agroalimentario se quiere?, ¿quién lo define y cómo?). Si se obvia el contexto en que se desarrolla la biotecnología, se simplifica su complejidad asociada, lo cual contribuye a perpetuar el distanciamiento entre posturas.

En el fondo de la controversia radica un desajuste fundamental entre el ritmo de la innovación científica y su ritmo de asimilación social. Mientras este desajuste persista, en el debate sobre los OMG agrícolas el resultado será la percepción de una política de hechos consumados alrededor de la cual se seguirá desarrollando un intercambio irreconciliable entre dos posturas enrocadas.

\section{Bibliografía}

Alonso, L. E. (1998). La mirada cualitativa en sociología. Madrid: Fundamentos. BARBER, B. (1984). Strong democracy: participatory politics for a new age. Berkeley, California: University of California Press.

BECK, U. (1998). La sociedad del riesgo. Hacia una nueva modernidad. Barcelona: Paidós.

CARR, S. (1999). European Union safety regulation of genetically-modified crops. Disponible en: http://www-tec.open.ac.uk/cts/bpg.htm

CE (1999). Libro Blanco sobre Seguridad Alimentaria. COM(1999)719. Bruselas, enero. 
- (2001a). Hacia una visión estratégica de las ciencias de la vida y la biotecnología. Documento de consulta. COM(2001)454 final. Bruselas, 04-09-2001.

- (2001b). Libro Blanco sobre Gobernanza Europea. COM(2001)428 final. Bruselas, 25-07-2001.

- (2001c). Comunicación de la Comisión sobre el Principio de Precaución. $\operatorname{COM}(2001) 1$. Bruselas, 02-02-2000.

- (2002). Hacia una visión estratégica de las ciencias de la vida y la biotecnología. COM(2002)27 final. Bruselas, 23-01-2002.

- (2003). Commission recommendation on guidelines for the development of national strategies and best practices to ensure the coexistence of genetically modified crops with conventional and organic farming. C(2003)2624. Bruselas, 23-07-2003.

CIS (2001). Opiniones y actitudes de los españoles hacia la biotecnología. Estudio 2412. Centro de Investigaciones Sociológicas, marzo-abril.

CORBETTA, P. (2003). Metodología y técnicas de investigación social. Madrid: McGraw Hill.

DE MARCHI, B.; FunTOWICZ, S. (2004). «La gobernabilidad del riesgo». En: LuJÁN, J. L.; ECHEVERRÍA, J. (eds.). Gobernar los riesgos. Ciencia y valores en la sociedad del riesgo. Madrid: Biblioteca Nueva.

DenZIN, N. K.; LinCOLN, Y. S. (eds.) (1994). Handbook of qualitative research. Thousand Oaks, Ca.: Sage.

Di MASsO, M. (2005). Gestión política del riesgo. La controversia sobre la agricultura $M G$ en Catalunya, en el marco de la regulación europea de transgénicos. Memoria de investigación. Doctorado en Sociología. UAB, septiembre.

ETZIONI, A. (1995). The spirit of community: rights, responsabilities and the communitarian agenda. Londres: Fontana Press.

EuROPEAN Opinion ResEARCH GROUP (EORG) (1991). Opinions of Europeans on biotechnology in 1991. EB 35.1, julio. Disponible en: http://europa.eu.int/comm/ public_opinion

EORG (1993). Biotechnology and Genetic Engineering. What Europeans think about it in 1993. EB 39.1, octubre.

- (1997). The Europeans and modern biotechnology. EB 46.1, julio.

- (2000). Europeans and modern biotechnology. EB 52.1, marzo.

- (2003). Europeans and biotechnology in 2002. EB 58.0, marzo.

- (2005). Europeans, Science and Technology. Special report. EB 63.1, junio.

- (2005). Social Values, Science and Technology. Special report. EB 63.1, junio.

FONT, N. (2001). El concepto de gobernanza. Ejercicio de titularidad, UAB. No publicado.

FunTOWICZ, S.; RAVETZ, R. (2000). La ciencia postnormal. Barcelona: Icaria.

GARCÍA MENÉNDEZ, P (2004). «Las estrategias de resolución de problemas y el estudio científico del riesgo: el caso de los alimentos transgénicos». En: LUJÁN, J. L.; ECHEVERríA, J. (eds.). Gobernar los riesgos. Ciencia y valores en la sociedad del riesgo. Madrid: Biblioteca Nueva.

GASKELL, G. y otros (2006). Europeans and Biotechnology in 2005: Patterns and Trends. A report to the European Commission's Directorate-General for Research. EB 64.3, mayo.

GIDDENS, A. (2004). Consecuencias de la modernidad. Madrid: Alianza Editorial.

- (2005). Un mundo desbocado. Los efectos de la globalización en nuestras vidas. Madrid: Taurus.

Habermas, J. (1999). Problemas de legitimación en el capitalismo tardio. Madrid: Cátedra. 
HARREMOËS, P. y otros (2002). The precautionary principle in the 20th Century. Late lessons from early warnings. Londres: Earthscan.

Hirst, P.; KhILnANI, S. (1996). Reinventing Democracy. Oxford, Cambridge: Blackwell Publishers.

KoOIMAN, J. (1993). Modern Governance. New government-society interactions. Londres: Sage.

LEMKOW, L. (1993). Public attitudes to genetic engineering: some European perspectives. European Foundation for the Improvement of Living and Working Conditions.

MANZini, E.; BigueS, J. (2000). Ecología y democracia. De la injusticia ecológica a la democracia ambiental. Barcelona: Icaria (Más Madera).

MAZUR, A. (1981). The dynamics of technical controversy. Washington DC: Communitation Press, Inc.

MuÑOZ, E. (1997). Nueva biotecnología y sector agropecuario. El reto de las racionalidades contrapuestas. IESA documento de trabajo 97-02. Madrid: Instituto de Estudios Sociales Avanzados.

Pateman, C. (1970). Participation and democratic theory. Cambridge: Cambridge University Press.

PEIX, J. (2004). «La nova agricultura catalana. La llei d'orientació agrària de 2001: un punt d'inflexió». En: ARGEMÍ, L.; RodRÍGUEZ, M. (coords.). L'agricultura moderna: de l'alimentació al medi ambient. Barcelona: Universitat de Barcelona.

PetTy, R. E.; HaugTVEDT, C. P.; SMITH, S. M. (1995). «Elaboration as a determinant of attitude strength: creating attitudes that are persisent, resistant, and predictive of behaviour». En: PETTY, R. E.; KROSNICK, J. A. (eds.). Attitude strength: antecedents and consequences. Mahwah: Lawrence Erlbaum Associates.

Pierre, J.; Peters, B. (2000). Governance, politics and the state. Londres: Macmillan Press Ltd.

Pindado, F.; Rebollo, O.; Martí, J. (2002). Eines per a la participació ciutadana. Bases, mètodes i tècniques. Diputació de Barcelona: Flor de Maig-Papers de Participació Ciutadana.

POORTINGA, W.; PidGEON, N. F. (2006). «Exploring the structure of attitudes towards genetically modified food». Risk analysis, 26, 6: 1-13.

Riechmann, J. (2004). Transgénicos: el haz y el envés. Una perspectiva crítica. Madrid: Los Libros de la Catarata.

RIECHMANN, J.; TICKNER, J. (coords.) (2002). El principio de precaución. En medio ambiente y salud pública: de las definiciones a la práctica. Barcelona: Icaria (Más Madera).

TȦbara, D.; Polo, D.; LemKow, L. (2003). «Precaución, riesgo y sostenibilidad en los organismos agrícolas modificados genéticamente». Politica y Sociedad, 40, 3: $81-103$.

TAIT, J. (2001). «More Faust than Frankenstein: the European debate about the precautionary principle and risk regulation for genetically modified crops». Journal of Risk Research, 4, 2: 175-189.

TODT, O. (2004). «El conflicto sobre la ingeniería genética y los valores subyacentes». Sistema, núm. 179-180, marzo. 